\title{
Carga contaminante de los residuos sólidos municipales en la cabecera departamental de Jalapa, Guatemala
}

\author{
Pollution load of municipal solid waste in the City of Jalapa, Guatemala
}

\author{
Gustavo Figueroa-Campos \\ Escuela Regional de Ingeniería Sanitaria y Recursos Hidráulicos (ERIS), Facultad de Ingeniería, \\ Universidad de San Carlos de Guatemala, Guatemala.
}

*Autor al que se dirige la correspondencia: gustavofigueroa84@gmail.com

Recibido: 28 de septiembre 2016 / Revisión: 26 de enero 2017 / Aceptado: 08 de mayo 2017

\section{Resumen}

Se expone la carga contaminante de los residuos sólidos municipales, en la cabecera departamental de Jalapa, Guatemala. Cada residuo, sin importar su origen o forma, tiene una composición química específica, la cual indicará su grado de contaminación o carga contaminante. El estudio se enfocó en el análisis de los residuos sólidos de tipo domiciliar, institucional, comercial, mercados y barrido y limpieza de calles por un periodo de cinco días. La muestra se analizó mediante la determinación de las fracciones de composición de los residuos, clasificándolos en: papel, cartón, plástico, pet, restos de comida, duroport, textiles, hule, vidrio, papel higiénico, pañales y madera. Para el cálculo de la carga contaminante se multiplicó los porcentajes que aporta el carbono, hidrógeno, oxígeno, nitrógeno y azufre a cada fracción de los residuos por el peso total de cada componente para luego dividirlo por el peso atómico. Para minimizar la carga contaminante de los residuos sólidos, la recolección debe darse con una frecuencia mínima de dos veces por semana, para evitar proceso de descomposición, pudiendo generar vectores y enfermedades en los sitios temporales de disposición que afecten a la población. El volumen de los desechos generados por el barrido de calles presenta la mayor carga contaminante, dando como resultado porcentajes altos en los componentes de carbono, hidrogeno, oxígeno y nitrógeno, sin embargo la misma puede reducirse implementando prácticas de valorización como el compostaje. Uno de los grandes problemas es el aporte de nitrógeno que en cantidades excesivas puede causar contaminación al suelo y a cuerpos de agua superficial.

Palabras claves: Composición física, composición química, educación sanitaria, recolección

\section{Abstract}

$\mathrm{T}$ The pollution load of municipal solid waste in the departmental capital of Jalapa is discussed. Each waste, regardless of their origin or form, has a specific chemical composition, which shall indicate the degree of pollution or pollution load. The study focused on the analysis of solid waste type household, institutional, commercial and street sweeping for a period of five days. The sample was analyzed by determining the fractions of waste composition, classifying them into paper, cardboard, plastic, pet, food scraps, styrofoam, textiles, rubber, glass, toilet paper, diapers and wood. To calculate the pollution load percentages provided by the carbon, hydrogen, oxygen, nitrogen and sulfur to each fraction multiplied by the total weight of each component and then divided by the atomic weight. To minimize the pollution load of solid waste, collection must occur with a frequency of twice a week, in order to avoid decomposition process, potentially leading to disease vectors and temporary disposal sites affecting the population. The volume of waste generated by street sweeping has the highest pollution load, resulting in high percentages of the carbon, hydrogen, oxygen and nitrogen components, but this can be reduced by implementing recovery practices such as composting. One of the big problems is the contribution of nitrogen that in excessive amounts can cause contamination to the soil and bodies of superficial water.

Keywords: Waste generation and characterization, chemical composition, sanitary education, waste collection 


\section{Introducción}

En la República de Guatemala y por ende en la cabecera departamental de Jalapa, área del estudio, se carece de lineamientos precisos para lograr una buena gestión o mejoras sustanciales en el manejo de residuos sólidos (Banco Interamericano de Desarrollo [BID], 2010). La cabecera departamental de Jalapa desde el 20 de abril de 2015 cuenta con un reglamento para la administración y funcionamiento del servicio municipal de recolección, tratamiento y disposición final de desechos y residuos sólidos, sin embargo un reglamento no garantiza la gestión integrada de residuos sólidos, por lo que se requiere un mayor compromiso de las autoridades locales para tratar esta problemática. Vale la pena destacar que un manejo inapropiado de los residuos sólidos conlleva un riesgo a la salud pública y al medio ambiente (BID, 2010).

Implementar mejoras o diseñar sistemas de manejo y tratamiento de residuos sólidos en una localidad, implica la necesidad de conocer las características de esos residuos, su tasa de generación, así como su composición y densidad, lo cual es indispensable para determinar el método de almacenamiento, recolección, tratamiento y disposición final más adecuados (Kunitoshi, 1983). Así mismo, la caracterización de los residuos sólidos puede contribuir significativamente al desarrollo de una legislación, regularizaciones, prácticas de manejo, proveer información necesaria para el mejoramiento del sistema de residuos sólidos e identificar, caracterizar y cuantificar la influencia de los componentes étnicos, religiosos y culturales en la producción de los residuos sólidos (Hristovski, Olson, Hild, Peterson, \& Burge, 2007). Cabe mencionar que la composición de los residuos sólidos está estrechamente relacionada con los ingresos económicos de la población y actividades sociales (Gómez, Meneses, Ballinas, \& Castells, 2009)

Cada residuo sólido tiene una composición física, química y biológica específica, la cual indicará los procesos de transformación que se han de seleccionar (Sáez \& Galbán, 2007). Excesivas concentraciones de componentes químicos pueden causar aumento considerable de nutrientes, disminuyendo así el oxígeno disuelto en los cuerpos de agua (Organización Panamericana de la Salud, 1996). Por eso es de suma importancia conocer las concentraciones químicas de los residuos sólidos y así poder determinar la carga contaminante de su composición.

Los componentes que se analizan para el cálculo de la carga contaminante de los residuos sólidos son el carbono, hidrógeno, oxígeno, nitrógeno y azufre.
Así mismo al analizar las cargas contaminantes de los residuos sólidos se deben tomar en cuenta todos los factores que pueden hacer variar dicha carga, principalmente el agua y tener cuidado con los porcentajes de los componentes.

El pH de los residuos sólidos de la cabecera departamental de Jalapa varían entre 5.0 y 6.0, debido a su estado ácido, los procesos de descomposición se dan más rápidamente, es por eso que la recolección debe darse con una frecuencia mínima de dos veces por semana y así evitar generación de vectores y proliferación de enfermedades en los sitios temporales de disposición (Figueroa \& García, 2014).

Implementar mejoras, diseñar sistemas de manejo y tratamiento o determinar la composición química de residuos sólidos en una localidad, implica la necesidad de conocer las características de esos residuos, su tasa de generación, así como su composición y densidad, lo cual es indispensable para determinar la carga contaminante de los mismos (Tchobanoglous, Theisen, \& Vigil, 1997).

La manera ideal de obtener esta información es a través de un estudio de caracterización de residuos sólidos (Brown et al., 2003), para lo cual se obtienen muestras de un determinado número de viviendas, que deberán ser representativas del universo de la población.

Los residuos sólidos contribuyen al efecto invernadero antropogénico, compuesto fundamentalmente por metano $\left(\mathrm{CH}_{4}\right)$, dióxido de carbono $\left(\mathrm{CO}_{2}\right)$ y óxido nitroso $\left(\mathrm{N}_{2} \mathrm{O}\right)$. La mayor contribución de gases de efecto invernadero se producen por procesos anaerobicos de descomposición (BID, 2010), lo que conlleva a la necesidad del manejo adecuado de los residuos sólidos.

Para el buen desarrollo e implementación de programas de manejo de residuos sólidos, se requiere la implementación de proyectos en educación sanitaria que involucre a la población, que refuerce el conocimiento y habilidades que se hayan adquirido en seminarios, cursos y capacitaciones. Por otra parte el manejo de los residuos sólidos, es en su mayoría, responsabilidad de los gobiernos locales. Esto es una tarea compleja, que requiere una adecuada organización y cooperación entre interesados del sector público y privado (Zarate, Slotnick, \& Ramos, 2008).

Es evidente la necesidad de planes de manejo de desechos sólidos, especialmente en los países en desarrollo (Wilson, Velis, \& Cheesemann, 2006), pero la falta de literatura que explora la aplicación actual de los sistemas de manejo de residuos sólidos, ha tenido como resultado la copia de planes de otros países. Como lo 
establece la investigación realizada por Marshall y Farahbakhsh (2013) es contraproducente para los países en desarrollo usar estrategias y políticas desarrolladas por países con alto ingreso; los acercamientos deben ser locales, críticos, creativos y tomados como propios por las comunidades.

\section{Materiales y métodos}

\section{Área de estudio}

La ciudad de Jalapa está localizada a 1,362 msnm, a una latitud de $14^{\circ} 38^{\prime} 02^{\prime \prime}$ y longitud $89^{\circ} 58^{\prime} 52^{\prime \prime}$, geográficamente limita al norte con Guastatoya (El Progreso); al este con San Pedro Pinula y San Manuel Chaparrón (Jalapa); al sur con San Carlos Alzatate, Monjas y Mataquescuintla (Jalapa); y, al oeste con Sanarate, Sansare (El Progreso) y Mataquescuintla (Jalapa) (Consejos Municipales de Desarrollo del Municipio de Jalapa \& Secretaría de Planificación y Programación de la Presidencia, 2010). La poblacional del área urbana de la cabecera departamental de Jalapa para el año 2014 era de 57,395 habitantes (Instituto Nacional de Estadística [INE], 2003).

\section{Procedimiento}

Los residuos sólidos analizados fueron los de origen institucional, comercial, barrido y limpieza de calles y domiciliar. La primera actividad fue el premuestreo, la cual se efectuó con el fin de determinar los valores de generación per cápita por día (PPC) de residuos sólidos, y estimar el número total de viviendas donde se colectarán las muestras. Para determinar el tamaño del premuestreo se utilizaron los criterios establecidos por la Norma Mexicana NMX-AA-61-1985, Protección al ambiente, contaminación del suelo, residuos sólidos municipales, determinación de la generación, (Secretaria de Comercio y Fomento Industrial, 1985), obteniéndose un total de 25 viviendas a premuestrear. Para la estimación de la generación per cápita preliminar se efectuó el pesaje de cada una de las bolsas obtenidas durante los cinco días de caracterización y se dividió por el número de habitantes promedio por

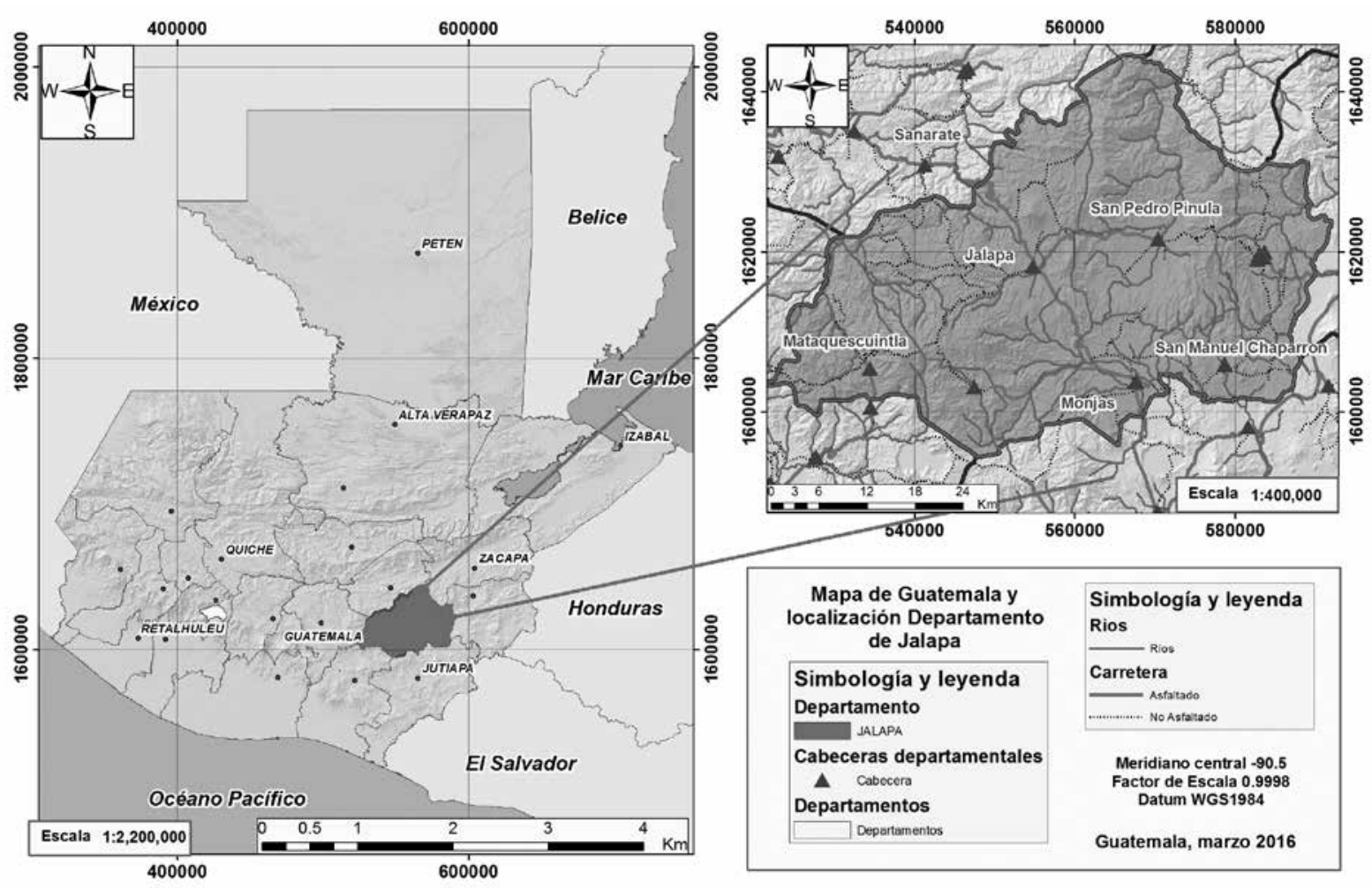

Figura 1. Cabecera departamental de Jalapa. 
vivienda, obteniendo una producción per cápita promedio de $0.42 \mathrm{~kg} / \mathrm{hab} /$ día.

La segunda actividad fue la determinación del tamaño real de la muestra, se utilizó la metodología propuesta por Kunitoshi (1983). La cual establece conocer la desviación estándar, valor que corresponde a la generación per cápita preliminar, $0.42 \mathrm{~kg} / \mathrm{hab} / \mathrm{día}$, error permisible que debido a posible sesgo en la participación de los domicilios y obtención de residuos no comunes durante el muestreo el valor oscila entre 1 y $15 \%$ de la PPC, se utilizó el 15\%, grado de confiabilidad 95\% y un número de casas para el municipio de Jalapa de 11,479 (INE, 2003), dando un total de 169 viviendas.

Para la caracterización de los residuos institucionales, se tomaron muestras provenientes del edificio de Gobernación Departamental, Policía Nacional Civil, Municipalidad, Presidio de hombres y mujeres. Las muestras de los residuos comerciales incluyeron el mercado municipal, comedores, abarroterías y tiendas de mayor producción en el municipio. Los residuos sólidos del barrido de calles se recolectaron de las calles principales de la cabecera municipal.

Como tercera actividad se realizó una caracterización de los residuos sólidos, utilizando el procedimiento propuesto por Kunitoshi (1983), se pesó el total de los residuos recolectados con la modificación, por conveniencia, de cinco días de recolección, posteriormente se colocaron en nailon y homogenizaron, separando la cuarta parte de los residuos y clasificándolos por categoría (Cantanhede, Sandoval, Monge, \& Caycho, 2005;
Kunitoshi, 1983). Esta metodología denominada prueba de composición física o método del cuarteo, relaciona el volumen de la muestra y tiempo de muestreo, obteniendo así representaciones adecuadas para la clasificación física de los residuos sólidos.

La última actividad fue el cálculo de la carga contaminante de cada elemento según su peso atómico (Tchobanoglous et al., 1997). Para esto se pesaron los residuos sólidos por categoría (Kunitoshi, 1983) (papel, cartón, plástico, pet, restos de comida, etc.) y se multiplicó por el peso atómico, la fórmula utilizada fue:

$$
\text { Moles }=\frac{\text { peso del elemento }}{\text { peso atómico }}
$$

Donde

Moles $=$ moles totales que aporta la categoría de residuo en carbono, hidrógeno, oxígeno, nitrógeno o azufre peso del elemento $=$ peso total de carbono, hidrógeno, oxígeno, nitrógeno o azufre

peso atómico = peso atómico de carbono, hidrógeno, oxígeno, nitrógeno o azufre

\section{Resultados}

La distribución de las categorías de los residuos sólidos de origen institucional, comercial, barrido de calles y domiciliar, refleja la variación en los pesos, la cual se debe al tipo de actividad que lleva a cabo el ente

Tabla 1

Composición de los residuos sólidos de la ciudad de Jalapa

\begin{tabular}{lcccc}
\hline Categorías & $\begin{array}{c}\text { Institucional } \\
(\%)\end{array}$ & $\begin{array}{c}\text { Comercial } \\
(\%)\end{array}$ & $\begin{array}{c}\text { Barrido calles } \\
(\%)\end{array}$ & $\begin{array}{c}\text { Domiciliares } \\
(\%)\end{array}$ \\
\hline Papel y cartón & 26.51 & 11.46 & 40.32 & 11.20 \\
Plástico & 7.69 & 7.64 & 12.90 & 10.06 \\
Pet & 7.69 & 4.58 & 6.45 & 1.83 \\
Restos de comida & 42.18 & 65.68 & 6.45 & 34.76 \\
Metales & 1.51 & 2.29 & 8.06 & 2.74 \\
Duroport & 2.98 & 0.76 & 3.23 & 1.83 \\
Textiles y hule & 0.00 & 1.53 & 3.23 & 14.94 \\
Vidrio & 1.51 & 1.53 & 3.23 & 4.27 \\
Papel higiénico & 9.94 & 4.54 & 0.00 & 18.37 \\
Otros & 0.00 & 0.00 & 16.13 & 0.00 \\
Total & 100 & 100 & 100 & 100 \\
\hline
\end{tabular}


generador. Como se puede observar en la Tabla 1 los restos de comida es la categoría que más peso presenta en la distribución, exceptuando el barrido de calles, donde se genera un mayor peso en papel y cartón.

En la Figura 2 se presentan los porcentajes totales de la generación de los residuos institucionales, comerciales, barrido de calles y domiciliares teniendo un mayor porcentaje en los restos de comida seguidos por el papel y cartón.

\section{Determinación carga contaminante}

En la Tabla 2 se muestra la carga contaminante de cada tipo de residuo en moles de carbono, hidrogeno, oxigeno, nitrógeno y azufre, con estos componentes podemos determinar el grado de contaminación que el residuo está generando al medio.
Tabla 2

Comparación de la carga contaminante de los diferentes tipos de residuos sólidos según su origen

\begin{tabular}{lrcrr}
\hline \multicolumn{5}{c}{ Carga contaminante (base húmeda) } \\
\hline $\begin{array}{l}\text { Componente } \\
\text { (mol) }\end{array}$ & D & I & C & BC \\
\hline Carbono & 31.97 & 45.64 & 30.14 & 94.83 \\
Hidrógeno & 49.29 & 71.35 & 47.11 & 146.05 \\
Oxígeno & 17.35 & 26.26 & 16.90 & 52.32 \\
Nitrógeno & 1.00 & 1.00 & 1.00 & 1.00 \\
Azufre & 0.06 & 0.09 & 0.07 & 0.11 \\
\hline
\end{tabular}

Nota. $\mathrm{D}=$ Domiciliares; $\mathrm{I}=$ Institucional; $\mathrm{C}=$ Comercial; $\mathrm{BC}=$ Barrido calles.

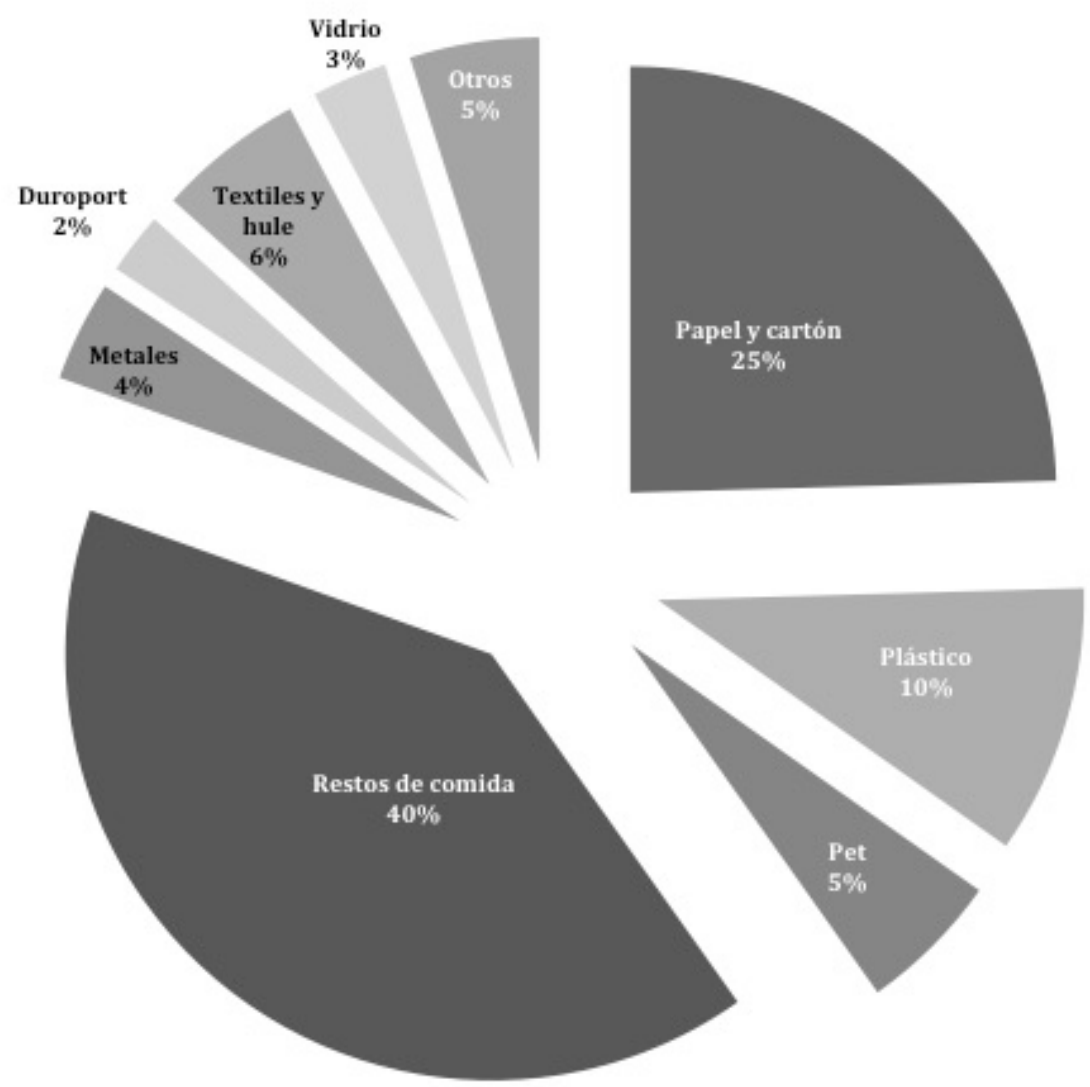

Figura 2. Composición porcentual de los residuos. 


\section{Discusión}

Jalapa presenta una generación de residuos sólidos per cápita de $0.66 \mathrm{~kg} / \mathrm{hab} /$ día, comparado con producciones per cápita tales como San Lucas Sacatepéquez, $0.45 \mathrm{~kg} / \mathrm{hab} /$ día (Domínguez \& Franco, 1996), Coatepeque, $0.51 \mathrm{~kg} / \mathrm{hab} /$ día (Gilroy, 2005) y Santa Catarina Pinula, $0.83 \mathrm{~kg} / \mathrm{hab} /$ día (Raudales \& Gradiz, 2012), podemos observar que Jalapa presenta una generación per cápita promedio y un comportamiento típico en la generación de residuos sólidos en áreas urbanas en municipios de Guatemala.

En el análisis realizado de la carga contaminante de cada tipo de residuos, se puede afirmar que los de origen del barrido de calles presenta la mayor carga contaminante, sin embargo, esto refleja la mala gestión integral de residuos sólidos y la falta de educación sanitaria promedio de la población de la cabecera departamental de Jalapa.

El comportamiento de la carga contaminante de los residuos de origen doméstico, comercial e institucional producen la menor contaminación al ambiente (Raudales \& Grádiz, 2012), sin embargo es necesario controlar la frecuencia de recolección de los desechos sólidos, para evitar procesos de descomposición que generen vectores y enfermedades en los sitios temporales de disposición, es necesario una recolección con una frecuencia mínima de dos veces por semana.

Los residuos reciclables como por ejemplo, papel, cartón, pet, restos de comida, vidrio, textiles y hule, presentes en un manejo de residuos sólidos son apropiados para recuperar y reusar. Ha sido estimado que el contenido reciclable varia de 13 al 20\% (Sharholy, Ahmad, Mahmood, \& Trivedi, 2008). Jalapa cuenta con los siguientes porcentajes valorizados: Domiciliar: papel y cartón $11.20 \%$, pet $1.83 \%$, restos de comida $34.75 \%$, vidrio $4.27 \%$ y textiles y hule $14.94 \%$. Comercial: papel y cartón $11.46 \%$, pet $4.58 \%$, restos de comida $65.68 \%$, vidrio $4.54 \%$ y textiles y hule $1.53 \%$. Institucionales: papel y cartón $26.51 \%$, pet $7.69 \%$, restos de comida $42.18 \%$, vidrio $9.94 \%$ en este caso sin presencia de textiles y hule.

Al realizar una valorización de las categorías arriba mencionadas se obtuvo una reducción en la carga contaminante, los porcentajes de reducción fueron los siguientes: Domiciliar: $21.72 \%$ carbono, $2.48 \%$ hidrógeno, $23.52 \%$ oxígeno y $0.94 \%$ azufre. Institucionales: $20.50 \%$ carbono, $20.07 \%$ hidrógeno, $18.97 \%$ oxígeno y 8.19 azufre. Comerciales: $23.92 \%$ carbono, $23.05 \%$ hidrógeno, $21.04 \%$ oxígeno y $4.38 \%$ azufre.
Para que las reducciones de los residuos susceptibles a valorización puedan implementarse efectivamente, como establece la investigación realizada por Burnley (2007) se requiere conocimiento del comportamiento de generación de los hogares, parte de esto incluye el comprender la variación que se tiene semana a semana en la generación a lo largo del año y esta área requiere de investigaciones en termino de estructuras a largo plazo.

Uno de los grandes problemas que presentan los municipios de Guatemala, es la disposición de los residuos sólidos en lugares inadecuados. El nitrógeno es un compuesto químico, que en grandes cantidades, puede afectar el suelo y los cuerpos de agua, causando contaminación al suelo y a cuerpos de agua superficiales (Sáez \& Galbán, 2007).

Por otra parte los riesgos a la salud para las personas que manipulan residuos sólidos con porcentajes elevados de carga contaminante son altos, debido al inadecuado manejo y falta de equipo de protección personal, dando como resultado el contacto directo con los residuos. Los principales riesgos son el contacto con vidrios rotos, heces fecales, residuos químicos, disolventes y agujas, entre las enfermedades más comunes están las deficiencias respiratorias, problemas de la piel, infecciones en los ojos y baja expectativa de vida. Los grupos más vulnerables son niños, mujeres y ancianos, que muchas veces juegan roles en actividades de reciclaje informal (BID, 2010).

\section{Agradecimientos}

A Adán Pocasangre por su contribución como mentor y asesor, a los catedráticos Pedro Saravia Celis, y Jorám Gil de la Escuela Regional de Ingeniería Sanitaria y Recursos Hidráulicos; asimismo, se reconoce el apoyo y la colaboración de Nery Adolfo Campos Polanco, Diagnóstico Radiológico (Dirad), Jalapa, a la Municipalidad de Jalapa, y personal del Laboratorio de Química y Microbiología Sanitaria de la ERIS en el desarrollo del estudio especial que generó el presente artículo.

\section{Referencias}

Banco Interamericano de Desarrollo. (2010). Informe de la evaluación regional del manejo de residuos solidos urbanos en America Latina y El Caribe. Washington, D.C.: Autor. 
Burnley, S. (2007). A review of municipal solid waste composition in the United Kingdom. Waste Management, 27(10), 1274-1285. doi: 10.1016/j. wasman.2006.06.018

Brown, D., Umaña, G., Gil, J., Salazar, C., Stanley, M., \& Bessalel, M. (2003). Guía para la gestión del manejo de residuos sólidos municipales. Enfoque: Centroamérica. San Salvador: Programa Ambiental Regional para Centroamérica.

Cantanhede, A., Sandoval, L., Monge, G., \& Caycho, C. (2005). Procedimientos estadísticos para los estudios de caracterización de residuos sólidos. Lima, Perú: Centro Panamericano de Ingeniería Sanitaria/Organización Panamericana de la Salud.

Concejo Municipal de Desarrollo del Municipio de Jalapa \& Secretaria de Planificación y Programación de la Presidencia. (2010). Plan de Desarrollo Municipal PD Jalapa, Jalapa. Guatemala: Autor.

Domínguez, V., \& Franco N. (1996). Manejo integral de los desechos sólidos en San Lucas Sacatepéquez, (Tesis de Maestría). Universidad de San Carlos de Guatemala, Escuela Regional de Ingeniería Sanitaria y Recursos Hídricos, Guatemala.

Figueroa, G., \& García, L. (2014). Gestión integral de residuos sólidos con énfasis en residuos susceptibles a valorización y disposición final, para la cabecera departamental de Jalapa (Tesis de Maestría). Universidad de San Carlos de Guatemala, Escuela Regional de Ingeniería Sanitaria y Recursos Hídricos, Guatemala.

Gilroy, L. (2005). Análisis de la situación del manejo de desechos sólidos municipales en el área urbana del municipio de Coatepeque (Tesis de maestría). Universidad de San Carlos de Guatemala, Escuela Regional de Ingeniería Sanitaria y Recursos Hídricos, Guatemala.

Gómez, G., Meneses, M., Ballinas, L., \& Castells, F. (2009). Seasonal characterization of municipal solid waste (MSW) in the city of Chihuahua, Mexico. Waste Management, 29(7), 2018-2024. doi: 10.1016/j.wasman.2009.02.006

Hristovski, K., Olson, L., Hild, N., Peterson, D., \& Burge, S. (2007). The municipal solid waste system and solid waste characterization at the municipality of Veles, Macedonia. Waste Management, 27(11), 1680-1689. doi: 10.1016/j. wasman.2006.09.003
Instituto Nacional de Estadística. (2003). Características de la población y de los locales de habitación censados, censos nacionales XI de población y VI de habitación. Guatemala: Autor.

Kunitoshi, S. (1983). Método sencillo del análisis de residuos sólidos. Lima, Perú: Organización Panamericana de la Salud/Centro Panamericano de Ingeniería Sanitaria.

Marshall, R., \& Farahbakhsh K. (2013). Systems approaches to integrated solid waste management in developing countries. Waste Management, 33(4), 988-1003. doi: 10.1016/j. wasman.2012.12.023

Organización Panamericana de la Salud. (1996). Análisis Sectorial de Residuos Sólidos en Colombia. Bogotá, Colombia: Autor.

Raudales, R., \& Grádiz, M. (2012). Modelo de gestión integral de residuos sólidos en el área urbana del Municipio de Santa Catarina Pinula (Tesis de Maestría). Universidad de San Carlos de Guatemala, Escuela Regional de Ingeniería Sanitaria y Recursos Hídricos, Guatemala.

Sáez, L., \& Galban M. (2007). Gestión Integral de los Residuos Sólidos. Matanzas, Cuba: Centro de Estudios de Medio Ambiente de Matanzas, Universidad de Matanzas "Camilo Cienfuegos".

Secretaria de Comercio y Fomento Industrial. (1985). Norma MX-AA-61-1985, protección al ambientecontaminación del suelo-residuos sólidos municipales-determinación de la generación. México, D.F: Autor.

Sharholy, M., Ahmad, K., Mahmood, G., \& Trivedi, R. (2008). Municipal solid waste management in Indian cities - A review. Waste Management, 28(2), 459-467. doi: 10.1016/j.wasman.2007.02.008

Tchobanoglous, G., Theisen, H., \& Vigil, S. (1997). Gestión integral de residuos sólidos. México, D.F: McGraw-Hill.

Wilson, D., Velis, C., \& Cheeseman, C. (2006). Role of informal sector recycling in waste managment in developing countries. Habitat International, 30, 797-808. doi: 10.1016/j.habitatint.2005.09.005

Zarate, M. A., Slotnick, J., \& Ramos, M. (2008). Capacity building in rural Guatemala by implementing a solid waste management program. Waste Management, 28(12), 2542-2551. doi: 10.1016/j.wasman.2007.10.016 